\title{
Contribution A La Connaissance Hydrogéologique Et Hydrogéochimique Des Aquifères De La Région De Beni-Hassan (Rif Septentrional, Maroc)
}

\author{
Redouan Alilouch \\ Karim El Morabiti \\ Abdelaziz El Mrihi \\ Université Abdelmalek Essaâdi, Département de Géologie, \\ Faculté des Sciences, Tétouan, Maroc
}

Doi: 10.19044/esj.2017.v13n30p441 URL:http://dx.doi.org/10.19044/esj.2017.v13n30p441

\begin{abstract}
The contribution to the hydrogeological, hydrochimical and bacteriological knowledge of the aquifers of Beni Hassan (Northern Rif) area constitutes one of the main aims of this study. This work aims: i) to make the inventory of water supply points of the area, ii) to study their relationship with the detrital and carbonated formations, as well as with the neighbouring some ones and iii) Clear the quality and the characteristics of the Chemical water type of these aquifers. The study area is located in the north of Morocco, in the septentrional Rif between the following lambert coordinates $\left(\mathrm{X}_{\mathrm{Min}}=501000 \mathrm{~m}\right.$ and $\left.\mathrm{X}_{\mathrm{Max}}=507000 \mathrm{~m}\right)$ and $\left(\mathrm{Y}_{\mathrm{Min}}=523000 \mathrm{~m}\right.$ and $\mathrm{Y}_{\mathrm{Max}}=$ $527000 \mathrm{~m}$ ). This latter belongs to the flyschs nappes (Mauritanian and massylien Flysch), the internal domain (Calcareous Dorsal) and external domain (Tanger Unit). The zone is characterized by two watersheds ("Wadi" watersheds Moulay Bouchta and "Wadi" watershed Martil), which is characterized in turn by aquifers of different lithological nature.
\end{abstract}

Keywords: Hydrogeochemistry; Mauritanian and massylien Flysch; Calcareous Dorsal; Chemical water type; Beni Hassan.

Résumé

L'étude porte sur la caractérisation hydrogéochimique, hydrogéologique et bactériologique des aquifères de la région de Beni Hassan (Rif septentrional) qui sont alimentés principalement par les eaux des précipitations, sous un étage bioclimatique subhumide et un hiver tempéré à chaud. Cette étude vise à : i) établir un inventaire des points d'eau de la région ; ii) étudier leurs rapports avec les formations géologiques (détritiques 
et carbonatées); et iii) dégager la qualité et les caractéristiques des faciès chimiques d'eau de ces aquifères. Le secteur objet de cette étude se situe au Nord du Maroc, dans le Rif septentrional entre les coordonnées $\mathrm{X}_{\mathrm{Min}}=$ $501000 \mathrm{~m} ; \mathrm{X}_{\mathrm{Max}}=507000 \mathrm{~m}$ et $\mathrm{Y}_{\mathrm{Min}}=523000 \mathrm{~m}$ et $\mathrm{Y}_{\mathrm{Max}}=527000 \mathrm{~m}$. Il fait partie du domaine interne (Dorsale calcaire), domaine externe (unité de Tanger) et domaine des flyschs de la chaîne rifaine (Flysch maurétanien et massylien). De point de vue hydrogéologique la zone d'étude se caractérise par deux bassins versants (BV): BV de l'Oued Martil et BV de l'Oued Moulay-Bouchta), qui se caractérisent à leur tour par des aquifères de nature lithologique différente.

Mots clés: Hydrogéochimie; Flysch maurétanien et massylien; Dorsale calcaire; Faciès chimique d'eau; Beni Hassan.

\section{Introduction}

L'eau c'est la source de vie, elle est également une source de maladie quand elle est polluée (Sohounou et al, 2017). Elle est beaucoup plus que juste un besoin humain ; c'est l'élément le plus essentiel pendant la vie (El Mountassir et $a l, 2017)$. Elle est devenue une question stratégique globale, dont la nécessité de la gestion s'intègre impérativement dans une perspective politique de développement durable (Bougarne et al, 2017 ; Garcia, 2006). Les réserves d'eau par habitant, qui encadrent déjà le seuil de l'effort avec $1000 \mathrm{~m}^{3} / \mathrm{hab} / \mathrm{an}$, seraient en 2020 environ $500 \mathrm{~m}^{3} / \mathrm{hab} / \mathrm{an}$ (Agoussine et Bouchaou, 2004).

Les eaux souterraines sont la plus grande et la plus importante ressource en eau en Afrique (Lapworth et al, 2017 ; MacDonald et al, 2012). Elle est souvent plus fiable, dans une proximité plus étroite aux utilisateurs, moins vulnérables à la pollution, et plus résilients à la variabilité de climat que l'eau de surface (Lapworth et $a l, 2013$, Lapworth et $a l, 2017$ ). Dans ce contexte, le Maroc dispose de ressources en eau relativement importantes. Il bénéficie en effet de nombreux atouts naturels: un château d'eau montagneux (la chaîne des Atlas) à larges espaces d'infiltration aux sommets enneigés et d'importantes nappes souterraines. Les eaux souterraines restent un facteur principal de développement socio-économique pour la plupart des pays méditerranéens (Baudron et al, 2014).

Les systèmes aquifères de la région de Beni Hassan, encore dite "El Hamra ", restent une source principale de l'eau pour l'irrigation et la subvention des besoins humains en eau potable. Ces dernières années, on a observé une baisse continue du niveau d'eau souterraine dans plusieurs secteurs. Ce phénomène expose la durabilité des ressources et donc l'économie entière de la région à un risque grave. En effet, l'exploitation d'eaux souterraines exige une connaissance détaillée des couches aquifères 
concernées (Mastrocicco et al, 2010 ; Tazioli et al, 2012 ; Giang et al, 2014), dont le but fondamental est la contribution à la connaissance hydrogéologique, hydrogéochimique et bactériologique des nappes aquifères de la région de Beni Hassan.

\section{Contexte géologique}

Le secteur objet d'étude se situe dans le domaine interne (Dorsale calcaire), le domaine externe (unité de Tanger) et le domaine des flyschs de la chaîne rifaine (Piqué, 1994, Suter, 1977, Wildi, 1983) (figure 1). Celui-ci se distingue par sa grande complexité structurale et par plusieurs contacts anormaux entre les unités géologiques (Durand Delga, 1972).

Figure 1: Schéma structural de la région d'étude avec localisation des points d'eau

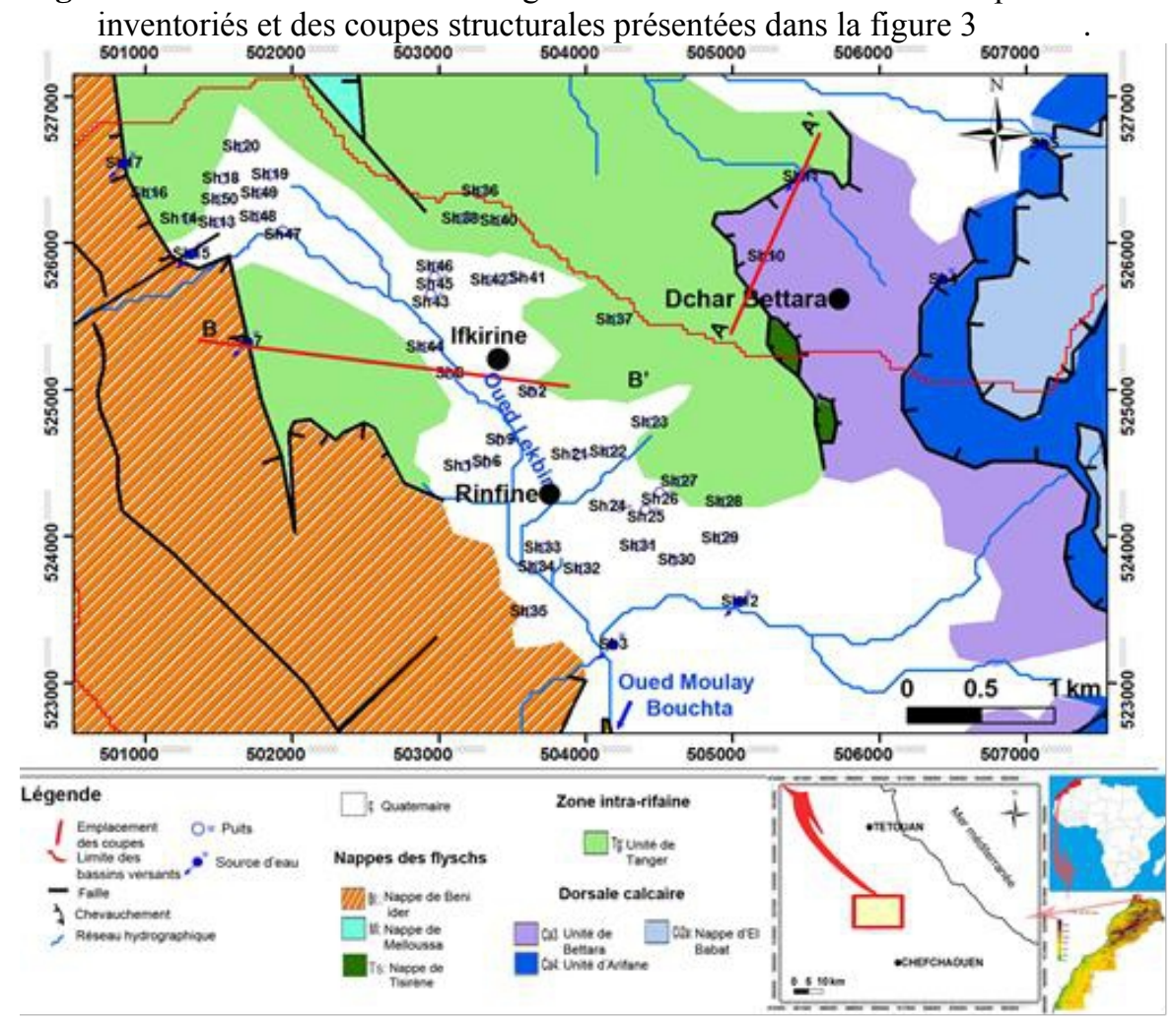

- Le domaine externe de la zone d'étude est formé par l'unité de Tanger qui est constituée de séries d'âge Crétacé supérieur (Marnes et minces lits de microbrèches en haut et des argiles, siltites et quelques bancs épais de calcilutite siliceuse passant au silex en bas). Il est largement charrié par les nappes des flyschs et le domaine interne (Didon, et al. 2004, Didon et Hoyez, 1978).

- Les nappes des flyschs (Flysch maurétanien et massylien) flottent sur les zones externes. Elles sont formées par la nappe de Beni Ider 
(calcarénites éocènes et grés-micacés oligocènes), la nappe de Melloussa (les séries sont caractérisées par des flyschs schistoquartziteux de l'Albo-aptien, des phtanites du Cénomanien et des marno-schistes du Crétacé supérieur) et la nappe de Tisirène, qui reposent par l'intermédiaire d'un contact tectonique plat ou à léger pendage vers l'Ouest et/ou l'Est sur les différents termes de l'unité de Tanger (Didon et Hoyez, 1978, Durand-Delga et al, 1960-1962, El Mrihi, 2005) ; cette dernière est affectée par des failles normales qui ont une direction N120 à N 140 à pendage vers le SW ou vers le NE (El Mrihi, 2005).

\section{Matériels et méthodes : Données et Matériels}

L'étude de la région est basée sur une carte topographique à l'échelle de 1/50.000 (Ministère de l'agriculture, 1970); deux cartes géologiques à l'échelle de 1/500.000 et de 1/ 50.000 (Suter, 1980a ; Didon et al, 2004); une carte structurale à l'échelle de 1/ 500.000 (Suter, 1980b); des données climatologiques et des coordonnées des stations météorologiques (Agence du Bassin Hydraulique de Loukkos de Tétouan).

\section{Méthodologie}

Les analyses chimiques et bactériologiques des eaux ont été réalisées au centre de Transfert des Technologies de l'eau (C.T.T) de l'agence du bassin hydraulique de Loukkos, au Laboratoire Régional de l'Environnement, au sein de la Faculté des Sciences Abdelmalek Essaâdi de Tétouan et dans la société Water Minéral à Chefchaoun. Ces analyses sont réalisées au laboratoire selon le protocole de l'analyse de Rodier (Rodier et $a l, 2009)$. La classification et la comparaison des eaux souterraines de la zone d'étude se basent sur des représentations graphiques, en s'appuyant sur les diagrammes de Piper et Schöeller-Berkaloff.

Le travail de cartographie a été mené par l'élaboration des cartes thématiques de la région d'étude et des coupes géologiques passant par différents points d'eau. Cette opération a été réalisée en se basant sur les données des cartes topographiques, géologiques et structurales comparées et confirmées par l'observation et l'étude structurale des différents faciès lithologiques et des systèmes aquifères sur le terrain.

\section{Résultats et discussion:}

\section{Caractéristiques climatiques}

Selon le quotient pluviométrique (Qp) ou indice climatique d'Emberger de la région $(\mathrm{Q} p=79,87)$ et la moyenne de la température 
minimale mensuelle $\left(\mathrm{T}_{\min }=7,5^{\circ} \mathrm{C}\right)$; la région d'étude est caractérisée par un étage bioclimatique subhumide et un hiver tempéré à chaud. En effet, les précipitations qui touchent les sommets des montagnes de la région peuvent dépasser les $1600 \mathrm{~mm}$, ce qui en fait l'une des zones les plus pluvieuses du Maroc (figure 2).

Plusieurs expressions empiriques introduisant les paramètres climatiques, ont été établies pour l'évaluation de l'évapotranspiration réelle (ETR) et/ou potentielle (ETP). Celles utilisées dans ce travail sont de Thornthwaite (1948), et de Turc (1955). L'évapotranspiration potentielle moyenne annuelle obtenue est de $680,51 \mathrm{~mm} / \mathrm{an}$; le maximum s'enregistre au mois de Juillet $(113,9 \mathrm{~mm})$ et le minimum au mois de Janvier $(17,71 \mathrm{~mm})$. L'évapotranspiration réelle annuelle (ETR) est estimée de 598,79 mm/an ; elle représente presque $86 \%$ des précipitations.

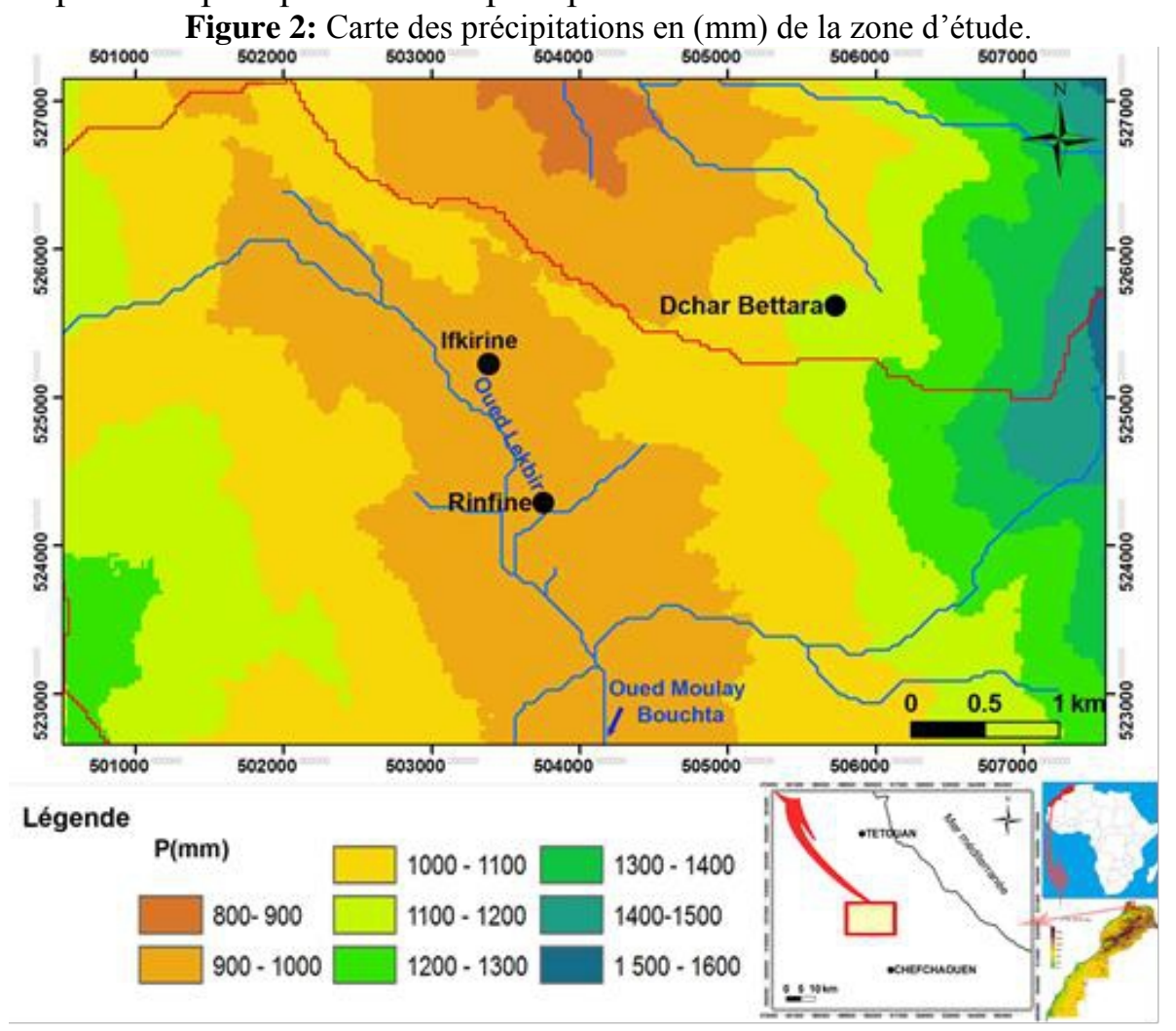

Pour les précipitations efficaces; les résultats obtenus à partir des données de la station pluviométrique "Nakhla" pour la période (1983-2000) par la méthode de Turc (Turc, 1955) donnent respectivement la moyenne de $95,81 \mathrm{~mm} / \mathrm{an}$, avec $13,79 \%$ de la pluviométrie moyenne annuelle. 


\section{Caractéristiques hydrogéologiques}

L'étude des relations d'émergence des différentes sources avec les contacts géologiques permet de mettre en relief le cadre hydrogéologique des aquifères du secteur d'étude en se basant sur des coupes structurales passant par les différentes sources d'eau. L'orientation de ces coupes structurales a été choisie de telle manière à faciliter le plus possible l'explication et l'analyse de leur émergence (figure 1).

Figure 3: Coupes structurales passant par les points d'eau Sh7, Sh8, Sh11et Sh10.
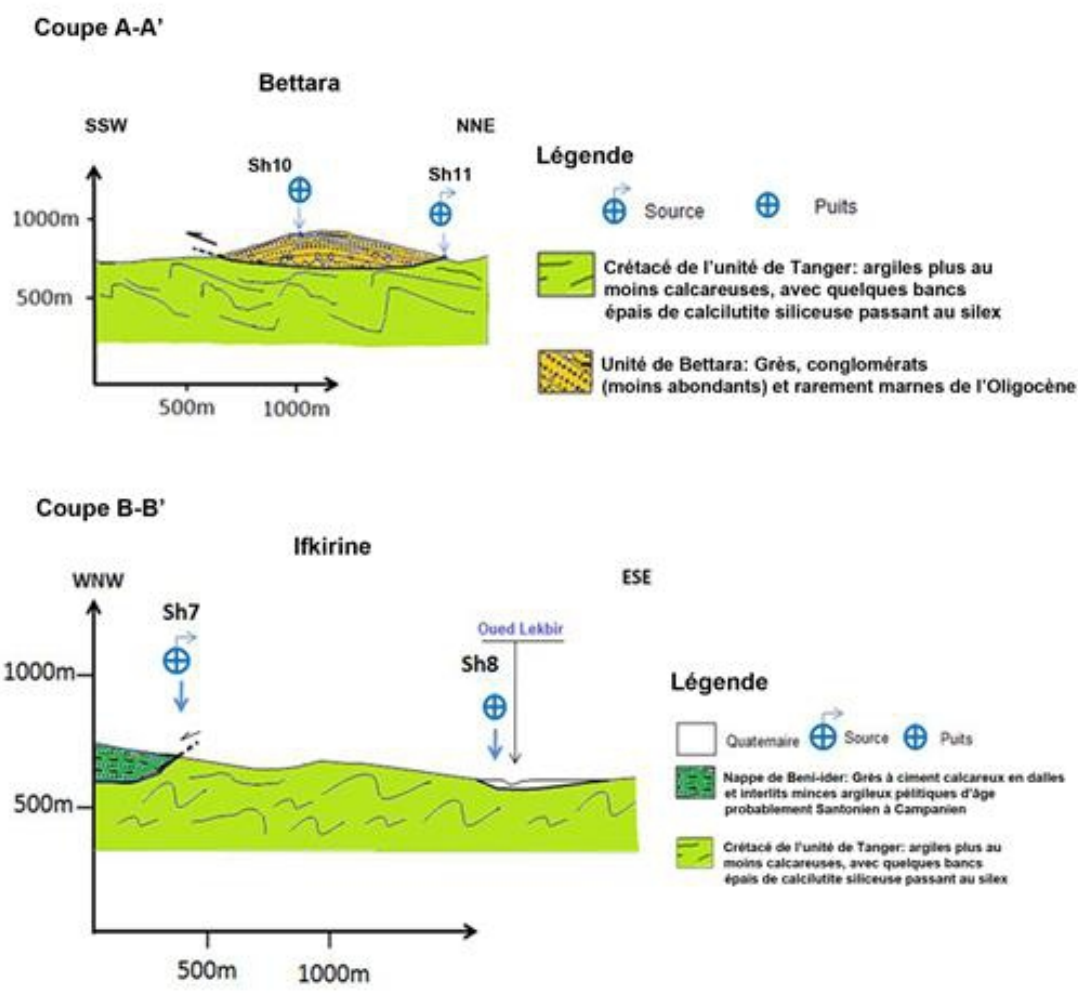

La région d'El Hamra où nous avons inventorié 8 sources et 42 puits est formée d'un aquifère de type poreux. Les sources d'eau de la région jaillissent au niveau des contacts anormaux (chevauchement): d'une part, entre la Dorsale calcaire (Unités de Bettara, d'Arifane et d'El Babat) et l'unité de Tanger. D'autre part, entre les flyschs de Beni Ider et l'unité de Tanger. Cette dernière est une formation argileuse plus au moins calcareuse avec quelques bancs épais de calcilutite siliceuse passant au silex, il s'agit d'une couche basale semi-imperméable à imperméable d'âge Crétacé supérieur, (Figures 1 et 3). 


\section{Carte piézométrique de l'aquifère à nappe libre de la région de Beni Hassan}

L'aquifere de la région de Beni Hassan est de type continu à nappe libre étendue. Il est caractérisé par une formation hydrogéologique perméable, homogène. Il est alimenté principalement par l'infiltration des précipitations efficaces.

Figure 4: Carte piézométrique de l'aquifère à nappe libre de la région de Beni Hassan.

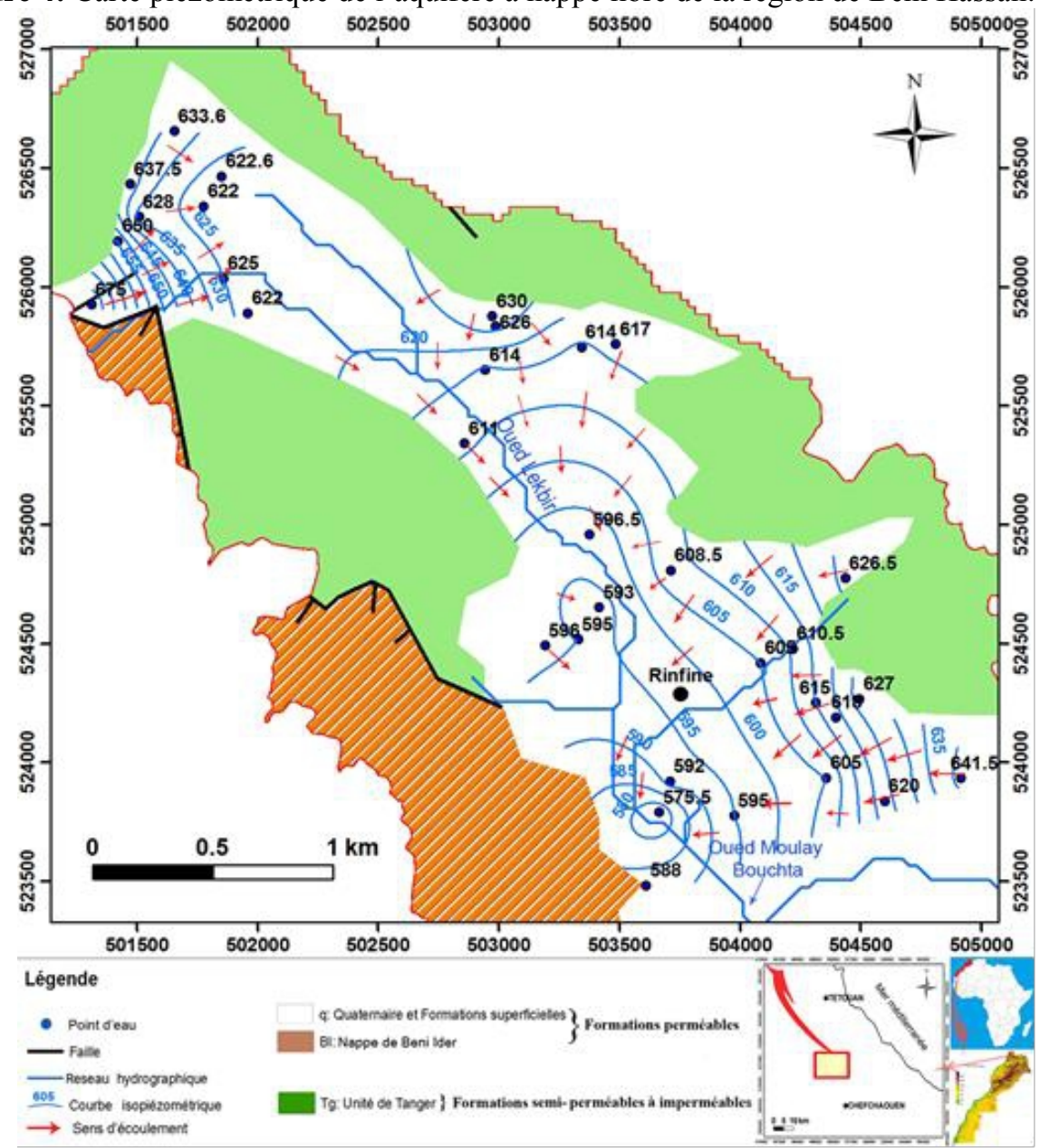

Le quaternaire de la région d'étude comporte 3 sources et 31 puits avec une profondeur d'eau qui varie entre 4 et $6 \mathrm{~m}$ (Tableau 1). La nappe d'eau est alimentée par les sources et les infiltrations d'eau de pluie (infiltration des précipitations efficace). La formation perméable de l'aquifère alluviale de la nappe libre du quaternaire constituée essentiellement par des matériaux détritiques (alluvions) : des sables, des graviers et des conglomérats d'âge Villafranchien supérieur et des éboulis (s.l). Il repose directement sur les flyschs de Bni Ider et aussi sur les 
formations de l'unité de Tanger (substratum imperméable). Ces dernières se comportent comme un aquiclude à aquitard dans certains cas.

Selon la carte piézométrique du quaternaire en basses eaux (Juin 2016) (figure 4); nous avons distingué l'existence des dépressions et des convergences piézométriques dues à l'exploitation intensive en deux points de pompage (Puits Sh9 et Sh34) et que le sens d'écoulement d'eau dans le secteur Nord est généralement orienté de l'Ouest vers l'Est, tandis que dans le secteur central, il est orienté du Nord et du Nord-Est vers le Sud et le SudOuest en alimentant les cours d'eau : Oued Moulay Bouchta et Oued Lekbir, (Tableau 1).

\section{Caractères physiques}

L'évolution de la conductivité d'eau mesurée sur place dans les points d'eau du secteur objet d'étude oscille (Tableau 1) entre les valeurs extrêmes: la valeur maximale est de $1942 \mu \mathrm{S} / \mathrm{cm}$ enregistrée dans le puits (Sh19) et la valeur minimale est de $346 \mu \mathrm{S} / \mathrm{cm}$ enregistrée dans la source (Sh45) avec une moyenne de $621.81 \mu \mathrm{S} / \mathrm{cm}$. Donc, les eaux de ces sources sont moyennement minéralisées (minéralisation comprise entre 500 et 1000 $\mathrm{mg} / \mathrm{L})$.

Le $\mathrm{pH}$ d'une eau naturelle dépend de la concentration en anhydride carbonique libre, entre autres composantes, et augmente avec la température. Pour la consommation humaine, l'organisation mondiale de la santé (O.M.S) admet pour limite minimale et maximale du $\mathrm{pH}$ des eaux souterraines 6,5 et 8,5 respectivement. Les 50 points d'eau montrent un $\mathrm{pH}$ normal, c'est-à-dire dans les normes, avec une moyenne de $\mathrm{pH}=7,8$. Les valeurs de $\mathrm{pH}$ sont cependant variables d'un point à un autre.

Tableau 1: Caractéristiques physiques des sources d'eau de la région de Beni Hassan.

\begin{tabular}{|c|c|c|c|c|c|c|c|c|c|c|c|}
\hline Point $d^{\prime} \operatorname{esp}$ & $\mathrm{pH}$ & $\mathrm{T}^{\circ} \mathrm{C}$ & $c 25^{\circ} \mathrm{C}$ & Nive a pitz ome trique $(m)$ & Debit (1 $1 \mathrm{x})$ & Points d'ean & $\mathrm{pH}$ & $\mathrm{T}^{\circ} \mathrm{C}$ & $c 25^{\circ} \mathrm{C}$ & Nivesu pienom etrigu o(m) & Debit(1s) \\
\hline $\mathrm{Shl}$ & \begin{tabular}{|l|l}
7.44 \\
\end{tabular} & 25.8 & 644 & 95 & - & $\operatorname{sh} 26$ & 8.33 & 1197 & 486 & 627 & - \\
\hline $\operatorname{sen} 2$ & 79 & 29 & 475 & 608.5 & - & $\$ 427$ & 3.21 & 16.5 & 707 & 645.5 & - \\
\hline $\operatorname{Sh} 3$ & 7.7 & 34 & 607 & 579 & 0.55 & Sb28 & 8.5 & 16 & 397 & 6585 & - \\
\hline $\operatorname{Sh} 4$ & 7.85 & 34 & 68.5 & 341 & 0.25 & $\sin 29$ & 7.39 & 15.6 & 677 & 641.5 & - \\
\hline $\mathrm{Su} 5$ & 7.78 & 13.2 & 358 & $9+1$ & 2 & $\operatorname{sh} 30$ & 8.14 & 16.4 & 496 & 623 & - \\
\hline Sh 6 & \begin{tabular}{|l|l} 
\\
\end{tabular} & 15 & 6.15 & 95 & - & \$k31 & 7.95 & \begin{tabular}{|l|l|}
15.7 \\
\end{tabular} & 457 & 600 & - \\
\hline $\mathrm{sh} 7$ & 7.2 & 12.7 & $\$ 158$ & 645 & 0.21 & $\operatorname{sk} 32$ & 7.7 & 15.3 & 903 & 598 & - \\
\hline $\mathrm{Sh} 8$ & 7.23 & 13.2 & 617 & 596.5 & - & $\operatorname{sk} 33$ & 8.5 & 16.5 & 609 & 592 & - \\
\hline $\sin 9$ & 7.13 & 34 & 613 & 598.3 & - & Sk34 & 7.5 & 19.2 & 740 & 575.5 & - \\
\hline Sh10 & \begin{tabular}{|l|l}
6.89 \\
\end{tabular} & 3 & 1132 & 78 & - & $\operatorname{sk35}$ & 7.98 & 141 & 576 & 598 & - \\
\hline shell & 6.62 & 13.8 & 1176 & $\$ 15$ & 0.18 & Sk36 & 7.37 & 19 & 547 & 621 & - \\
\hline $\operatorname{su} 12$ & \begin{tabular}{|l|}
7.4 \\
\end{tabular} & 34 & 507 & 651 & 0.8 & Sk37 & 7.68 & 167 & 435 & 672.5 & - \\
\hline She.13 & 7.32 & 15 & 536 & 650 & - & $8 k 38$ & 8.37 & \begin{tabular}{|l|}
16 \\
\end{tabular} & 599 & 641 & - \\
\hline She 14 & 7.22 & 21.8 & 535 & 667 & - & $\operatorname{sk} 39$ & 7.66 & 16 & 512 & 645.5 & - \\
\hline \$h 15 & 7.21 & 13.9 & 2,25 & 65 & 0.13 & $8 \mathrm{~b} 40$ & 7.72 & 19 & 431 & 635 & - \\
\hline Sh16 & \begin{tabular}{|l|}
.05 \\
\end{tabular} & \begin{tabular}{|l|}
259 \\
\end{tabular} & 598 & 704.5 & - & $\mathrm{sk41}$ & 3.04 & \begin{tabular}{|l|l|}
15.7 \\
\end{tabular} & 539 & 617 & - \\
\hline 83617 & 7.64 & 34.1 & 607 & 736 & fabis & $\mathrm{sk42}$ & 7.8 & 19.5 & 709 & 624 & - \\
\hline Sh 18 & 7.95 & 22 & 684 & 637.5 & - & $\mathrm{Sb} 43$ & 8.3 & 149 & 452 & 624 & - \\
\hline Sh19 & \begin{tabular}{|l|l}
8.39 \\
\end{tabular} & 25.2 & 1942 & 622.6 & - & sk44 & 8.21 & 148 & 427 & 611 & - \\
\hline $\sin 20$ & \begin{tabular}{|l|l}
8.46 \\
\end{tabular} & 15 & 662 & 633.6 & - & Sk45 & 8.4 & 17 & 346 & 625 & - \\
\hline $\operatorname{sen} 21$ & 8 & \begin{tabular}{|l|}
25.1 \\
\end{tabular} & 700 & 605 & - & Sb45 & 8.01 & 166 & 391 & 630 & - \\
\hline $\sin 22$ & 7.85 & 202 & 495 & 620.5 & - & sk47 & 7.62 & \begin{tabular}{|l|l|}
15.4 \\
\end{tabular} & 5.63 & 62 & - \\
\hline 8.23 & 7.66 & 29.7 & 456 & 625.5 & - & sh48 & 7.62 & 149 & 547 & 628 & - \\
\hline Sh 24 & 8.3 & 2.7 & 390 & 6.5 & - & Sk49 & 8.04 & 19 & 396 & 62 & - \\
\hline 8675 & 8. & 751 & 917 & 618 & - & $86=0$ & 9 & 147 & 875 & 628 & - \\
\hline
\end{tabular}


La valeur la plus élevée enregistrée est de 1'ordre de 8,5 dans le puits (Sh28); la valeur la plus basse mesurée est de 6,62 propre à la source (Sh11).

Le débit des sources d'eau de la région d'étude varie de faible (source Sh17) à fort; avec un maximum de 2 1/s enregistré à la source (Sh5). Tandis que, la température enregistrée des points d'eau oscille entre un minimum de $13.2^{\circ} \mathrm{C}$ et un maximum de $17^{\circ} \mathrm{C}$ (Tableau 1 ).

\section{Eléments chimiques majeurs et mineurs}

Les données du tableau 2 montrent que les bicarbonates dominent les anions dont les concentrations moyennes en eau des sources sont de l'ordre de $305.92 \mathrm{mg} / \mathrm{l}$ et le cation dominant est le calcium avec la valeur la plus élevée enregistrée est de l'ordre de $116.23 \mathrm{mg} / \mathrm{l}$ dans le puits (Sh4) et la valeur la plus basse observée est de l'ordre de $60 \mathrm{mg} / \mathrm{l}$, propre à l'eau de la source (Sh9).

Tableau 2: Concentrations en éléments chimiques d'eau des points d'eau types en (mg/l) de Beni Hassan.

\begin{tabular}{|c|c|c|c|c|c|c|c|c|}
\hline Points d'eau & $\mathrm{Ca}^{2-}$ & $\mathrm{Mg}^{2-}$ & $\mathrm{Na}^{-}$ & $\mathbf{K}^{-}$ & $\mathrm{HCO}^{-}$ & $\mathbf{C l}$ & SO${ }^{2-}$ & NO$^{-}$ \\
\hline Sh1 & 84.17 & 15.79 & 28.2 & 1.7 & 288.66 & 35.08 & 49.75 & 0 \\
\hline Sh2 & 80.18 & 10.94 & 22.4 & 1.2 & 290.79 & 22.35 & 17.63 & 0 \\
\hline Sh3 & 112.22 & 10.94 & 11 & 0.8 & 354.3 & 28.99 & 14.38 & 0 \\
\hline Sh4 & 116.23 & 8.51 & 12 & 0.9 & 350.05 & 32.9 & 12.13 & 0 \\
\hline Sh5 & 64.13 & 25.51 & 3.5 & 0.6 & 286.71 & 25.08 & 10.63 & 0 \\
\hline Sh6 & 78.18 & 14.58 & 19.8 & 1.8 & 285 & 29.11 & 20.2 & 0 \\
\hline Sh7 & 89 & 16.51 & 20.12 & 1.4 & 255.2 & 58.8 & 15.04 & 0 \\
\hline Sh8 & 71 & 12.84 & 19.4 & 1.5 & 235.3 & 29.61 & 21.3 & 0 \\
\hline Sh9 & 60 & 18.3 & 19.7 & 1.2 & 215.3 & 28.69 & 33.27 & 0 \\
\hline Sh10 & 114 & 12.28 & 21 & 1 & 321.16 & 55.6 & 8.49 & 0 \\
\hline Sh11 & 115 & 12.89 & 19.5 & 2.2 & 312.6 & 56 & 22 & 0 \\
\hline Sh12 & 79.54 & 10.09 & 10.4 & 0.7 & 238.32 & 29.2 & 21 & 0 \\
\hline
\end{tabular}

\section{Faciès d'eau}

Figure 5: Diagramme triangulaire de Piper (b) et semi- logarithmique de Schöellerberkaloff (a) représentant les faciès d'eau de la région El Hamra.

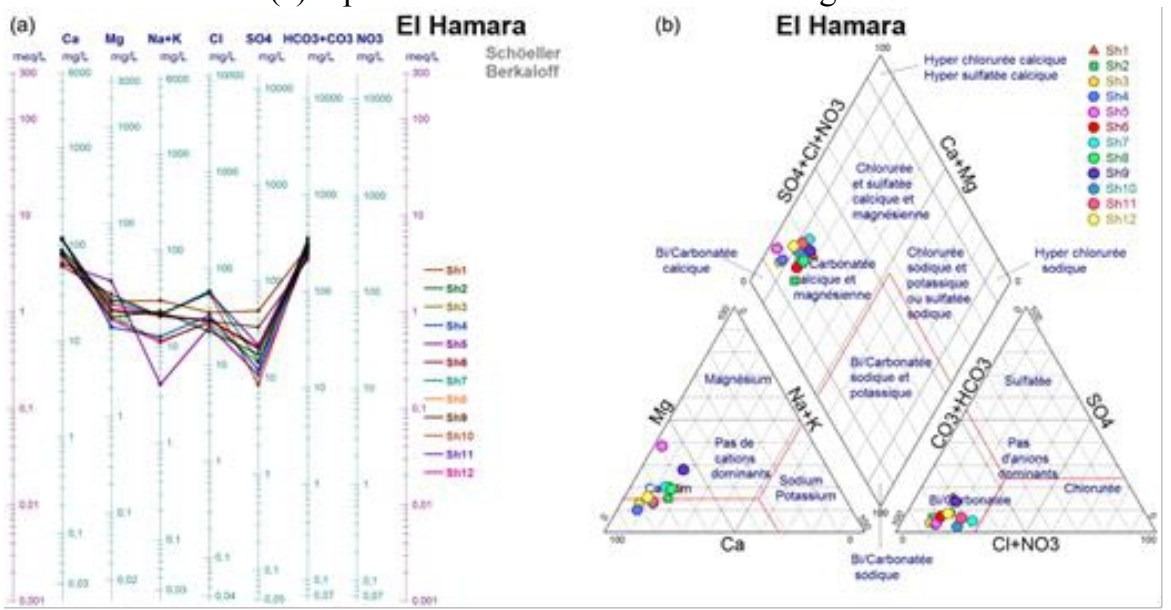


Les représentations graphiques de Piper (Chadha, 1999, Piper, 1944) et Schöeller-berkaloff (figure 5), affirment que les eaux souterraines de la zone ont une dominance de faciès bicarbonaté-calcique et magnésien.

\section{Dureté d'eau et caractéristiques bactériologiques}

La dureté d'eau $(\mathrm{TH})$ des sources et des puits de la commune El Hamra (Beni Hassan) oscille entre (Figure 6), $(4,4<\mathrm{TH}<6,6)$ assez dure, et $(6,6<\mathrm{TH}<8,4)$ dure. Généralement ces sources caractérisées par des eaux exemptes de nitrate, possèdent une bonne minéralité à la fois pures et légères.

Figure 6: Histogrammes de Figure 7: Nombre des Streptocoques fécaux et dureté des points d'eau. des Coliformes dans les points d'eau.

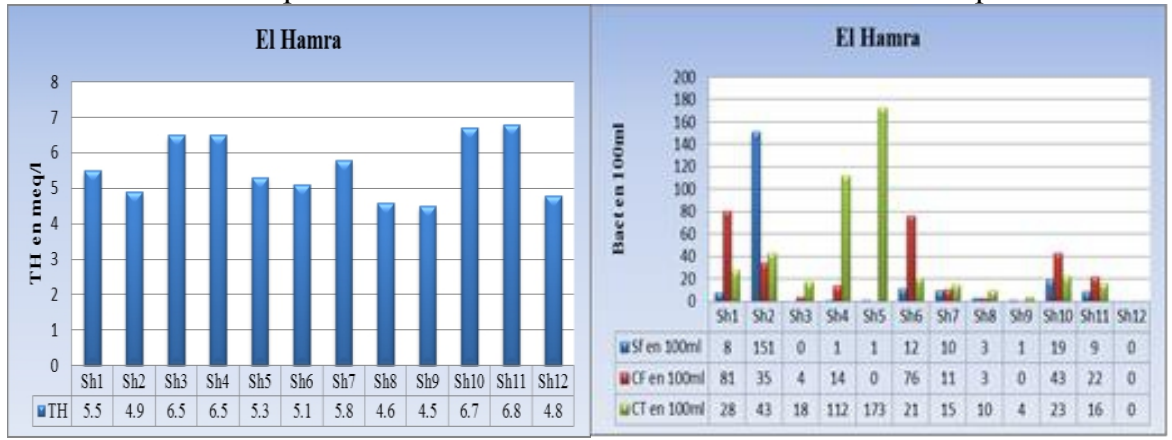

Les résultats des analyses bactériologiques des points d'eau révèlent la présence des germes indicateurs de contamination fécale (figure 7) ; cette contamination de ces points d'eau types ne dépasse pas les normes sauf dans quelques puits et sources qui sont abandonnés en plein air et utilisés uniquement pour l'irrigation des champs de culture parcellaires. Généralement ces données révèlent que les aquifères du secteur objet d'étude ont un pouvoir épurateur important.

\section{Conclusion}

Les aquifères de la région de Beni Hassan se caractérisent par leur nature lithologique différente d'une zone à l'autre. Elles ont un comportement hydrobiologique important. Les sources d'eau sourdent soit au niveau d'un contact anormal entre deux formations géologiques : une, correspondante à un aquifère au sommet et une autre aquiclude formant le socle; soit au niveau des failles normales affectant la formation aquifère. Elles sont de type pérenne et se caractérisent par un débit généralement moyen à faible. Les faciès de l'eau de la région d'étude sont à dominance bicarbonatée calcique et magnésienne. Généralement, l'eau de la région est assez dure à dure, de bonne qualité, exempte de nitrates et possède une bonne minéralité à la fois pure et légère. Ces points d'eau de la région jouent 
un rôle primordial à la subvention des besoins en eau potable, en irrigation et en usage domestique.

\section{References :}

1. Agoussine M., Bouchaou L., (2004). Les problèmes majeurs de la gestion de l'eau au Maroc. Science et changements planétaires / Sécheresse, 15 (2): 94-187.

2. Baudron P, Barbecot F, Aróstegui J.L.G, Leduc C, Travi Y, Martinez-Vicente D, (2014). Impacts of human activities on recharge in a multilayered semiarid aquifer (Campo de Cartagena, SE Spain).Hydrol.Process. 28, 2223-2236. doi:10.1002/hyp.9771.

3. Chadha, D.K. (1999). A Proposed New Diagram for Geochemical Classification of Natural Waters and Interpretation of Chemical Data. Hydrogeology Journal, 7: 431-439.

4. Didon J., Hogga A., \& Haddane M., (2004).Carte géologique: Souk Larbaa Beni Hessane (Echelle 1/50000). Serv. Géol. Maroc.

5. Didon, J. \& Hoyez, B., (1978). Les séries à faciès mixte, numidien et gréso-micacé, dans le Rif occidental (Maroc). C. R. somm. Soc. géol. France, 6 : 304-307.

6. Durand Delga M., Hottinger L., Marcais J., Mattauer M., Milliard Y. et Sutter G., (1960-1962). Données actuelles sur la structure du Rif. Carte structurale. Mem. Hrs. Serv. Soc. Géol. Fr. : 399-422.

7. Durand Delga M., (1972). La courbure de Gibraltar, extrémité occidentale des chaînes alpines unit l'Europe et l'Afrique. Ecl. Geo. Helv. 2 : 267-278.

8. EL Mountassir R., Bennani B., Merzouki H., Benjelloun Touimi G., Boumchita S., Benjelloun Y. \& Lahrichi A., (2017).Characterization of the chemical and bacteriological risks of the effluents from some services of the Hassan II Hospital Center in Fez. J. Mater. Environ. Sci. 8: 2288-2295

9. El Mrihi A., (2005).Structure et cinématique de mise en place des nappes de flysch mauritaniens (Rif externe nord occidental): Elaboration d'un modèle, thèse de Doct. Fac. Sci. Tétouan. Maroc: 269.

10. Garcia A., (2006). Etude de la dynamique des Escherichia coli dans les rivières du bassin de la Seine. Thèse Doctorat, Université Libre de Bruxelles : 15-16.

11. Lapworth D.J., MacDonald A.M., Tijani M.N., Darling W.G., Gooddy D.C., Bonsor H.C.\& Araguás-Araguás L., (2013).Residence times of shallow groundwater in West Africa: implications for hydrogeology and resilience to future changes in climate. Hydrogeol J. 21(3): 673-686. 
12. Lapworth D. J.,Nkhuwa D. C. W., Okotto-Okotto J., Pedley S., Stuart M. E., Tijani M. N.\& Wright J.,(2017). Urban groundwater quality in sub-Saharan Africa: current status and implications for water security and public health. Hydrogeol J: 1-24.

13. MacDonald A.M., Bonsor H. C., Dochartaigh B. E.O.\& Taylor R. G., (2012). Quantitative maps of groundwater resources in Africa. IOPscience. Environ Res Lett. Vol. 7: 7.

14. Mastrocicco M, Vignoli G, Colombani N, Zeid N.A (2010). Surface electrical resistivity tomography and hydrogeological characterization to constrain groundwater flow modeling in an agricultural field site near Ferrara (Italy). Environ EarthSci 61, 311-322. doi: 10.1007/s12665-009-0344-6.

15. Ministère de l'Agriculture et de la Réforme Agraire, (1970). Carte topographique: Souk Larbaa Beni Hassane (Echelle 1/50000). Rabat. Maroc.

16. Piqué A., (1994). Géologie du Maroc. Les domaines régionaux et leur évolution structural. éd. Pumag, Rabat, : 284.

17. Piper, A.M., (1944). A graphic procedure in the geochemical interpretation of water analyses. American Geophysical Union. Papers, Hydrology: 914-923.

18. Rodier, J., Bazin, C., Broutin, J.P., Chambon, P., Champsaur, H. \& Rodi, L., (2009). Water analysis, 9th edit. Dunod, Paris, France : 1579.

19. Suter, G., (1980a et b). Carte géologique et structurale de la chaîne rifaine au 1/500.000. Notes et Mémoires. Serv. Géo. Rabat. Maroc. $\mathrm{n}^{\circ} 245 \mathrm{a}$ et $\mathrm{b}$.

20. Sohounou, M., Vissin, E.W., Sintondji L.O. Houssou, C.S., \& Agbossou K. E. (2017). Physico-Chemical and Microbiological Qualities of Water From Wells, Drillings and Tanks Used as Drinking Water in the Municipality of Allada (Benin, West Africa). European Scientific Journal: 13(15).1857-7431.

21. Thornthwaite C.W., (1948). An approach toward a rational classification of climate. Géol. Review, 38 : 55-94.

22. Turc L. (1955). Le bilan d'eau des sols: relation entre la précipitation, l'évaporation et l'écoulement. Ann. Agron. : 5-131.

23. Wildi W., (1983). La chaîne tello-rifaine (Algérie, Maroc, Tunisie): structure, stratigraphie et évolution du Trias au Miocène. Rev. Géol. Dyn. Géogr. Phys., 24, (3): 201-297. 